\section{LIGATURE OF THE INNOMINATE ARTERY.}

BY BENNETT MAY, B.S., F.R.C.S.,

SURGEON TO THE QUEEN'S HOSPITAX, DEMONSTRATOR OF OPERATIVE SURGERY AT QUEEN'S COLLEGE, BIRMINGHAM.

AN annotation in THE LANCET of April 3rd favoured with special notice the case in which, seven days previously, I had tied the innominate artery for aneurysm. The patient was then doing well, but died from secondary hæmorrhage on the nineteenth day. This further record is from the bedside notes of our house-surgeon, Mr. A. B. Bennett.

J. $\mathrm{N}-$, aged thirty, a brewer's labourer, was admitted into the Queen's Hospital on March 3rd, 1886, suffering from a large aneurysm of the right subclavian artery. His occupation had exposed him for a long term of years to great exertions and violent straining, as in carrying heavy sacks on his shoulder, and the joint of this side had been dislocated on three occasions. He had never had syphilis or rheumatism, and had been a very moderate drinker. The first symptom of the disease appeared about seventeen months previously, in the form of shooting pains down the arm and forearm, with occasional numbness of the limb. but, in spite of increasing suffering, he struggled on with his work, doing most of it with the other arm. He discovered a local swelling above the clavicle about nine weeks before admission. This steadily and rapidly grew in size, and with it the pain became more severe. He then came under the care of Mr. W. B. Sparrow, of Burton-on-Trent, who diagnosed the aneurysm and sent him to me for its treatment.

March 5th.-The tumour lies just above the inner half of the clavicle; it measures three inches in the transverse and two inches and a half in the vertical direction. On the inner side it disappears under the sterno-mastoid; externally it reaches nearly to the acromion. The common carotid artery is carried inwards towards the middle line of the neck, and can be felt distinct from the tumour, over which it can be made to roll. Pulsation in the tumour is very strong, but bruit is either extremely faint or absent. There is a pulsation in the episternal notch, obviously that of the root of the common carotid, which is prominent here. Pressure effects on the brachial plexus are marked by intense pain with numbness down the arm and forearm and wasting of the muscles. The pain, which at times is excruciating and causes extreme restlessness, appears to affect mostly the peripheral area of the musculo-spiral nerve. There is no odema or venous engorgement of the limb, but the nails are slightly clubbed. The brachial artery, which is very superficial above the bend of the elbow, is no larger in calibre than a No. 3 catheter. The radial pulse is distinctly smaller than on the other side. All the superficial arteries are slightly fibroid. The right pupil is much smaller than the left. No laryngeal or pharyngeal symptoms exist. The chest was exannined by Dr. Suckling, who found no evidence of disease of the heart or of the large bloodvessels.

Treatment.-The man was placed on Tufnell's aneurysm diet, consisting of ten ounces of light dry solid food with eight ounces of fluids daily. He would not keep recumbent, owing to the severity of his pain, which caused him to sit up in bed and roll his arm about a great deal. Hypodermic injections of morphia in frequent half-grain doses seemed almost powerless to relieve him at first. Ordered ten grains of iodide of potassium three times a day.-11th: The pain is somewhat less, the morphia being more efficacious in giving relief. Aneurysm the same.18th: Condition much as before, but the patien's is wearying of the diet, and he is thinner and weaker.-25th: The slight improvement which was at first apparent has not been sustained. Pulsation is as strong as ever, and its superficial area is much larger, while the pain is as severe as at any previous time. He is clamorous for food, and not willing to continue the restricted diet any longer.

Under these circumstances $\dot{I}$ determined upon operative treatment, and therefore abandoned all medicine and placed him on a liberal dietary for a few days. After carefully considering all the circumstances of the case, together with the results of recent experience in operations on large arteries, there appeared to me no ground for excluding our most direct and certain method of procedure-viz., the Hunterian -by ligature of the innominate artery. Accordingly or March 27th, the patient being brought well under chloroform, I made an incision along the inner third of the claricle, and another down the line of the common carotid artery, 80 as to meet the first in the episternal notch. This skin flap was then dissected up, presenting veins tied and divided, the sternal with part of the clavicular origin of the sternomastoid cut across, and the sterno-hyoid and thyroid muscles also divided. The carotid sheath was then arrived at and opened on its inner side low in the neck. Proceeding down this artery the innominate was found. It appeared a vessel of unusually large dimensions, and was rather superficial. It seemed to be nearly an inch in diameter, but showed no local or fusiform expansion. Compression made against the sternum by a finger behind the vessel was found to control the pulsation both in the tumour and the carotid. I could not define its bifurcation nor make any approach to the first portion of the subclavian, as the structures over this vessel were matted and could hardly be identified. I afterwards found that it was flattened out over the aneurysmal sac. It was rather an arduous task to get the needle round the vessel; the difficulty was partly owing to. its size, but mainly to the existence of adhesions binding the vessel to the sternum and the parts on its outer side. The needle used was an old-fashioned silver one, broad, and well rounded at the point, with a large eye, and sufficiently flexible to take any desired curve. This was passed satisfactorily round the vessel, and then threaded with a small cord, to which a strip of ox aorta material, kindly sent me by Mr. Barwell, was attached, and by it pulled through. In tightening the tape, I had todraw the ends with very considerable force to stop the pulsation, the vessel offering great resistance and pulsating with great force. Just at the critica moment, however, the material gave way and broke across and a second piece introduced in a similar way suffered the same fate. I then endeavoured to imitate the principle of the flat ligature by using a cord made up of five or six mediumsized threads of catgut. This bore the strain very well, and after tightening with sufficient force to completely stop pulsation in the tumour and branches of the carotid, I drew on the ends still further, to allow of some subsequent relaxation in fixing the knot. At the same time I endeavoured to avoid crushing the coats of the artery. The ligature was secured with a third knot and cut short; the wound was dried from all clot, its edges brought partly together, a tube and strip of antiseptic gauze being left in one corner for drainage. The whole proceeding had been conducted under the spray and careful Listerian antiseptic details. The wound was then well padded, and covered with a gauze dressing secured by an elastic roller. On leaving the table no pulse was perceptible at the wrist or in the aneurysm. Four hours afterwards there was a faint pulse at the wrist. He had not much pain, but was restless from heat. Temperature normal; pulse 102; respiration 22. Had rallied well from the operation.

March 28th.-Seems very comfortable except for pain and burning of the arm. Temperature $98^{\circ} 8^{\circ}$; pulse (evening) 106; respiration 22. A small thready pulse can be felt at the wrist and also in the superficial temporal.-29th: Wound dressed to-day; thoroughly aseptic; tube removed, and drainage effected by a strip of antiseptic gauze carried to the bottom. This being the first occasion of exposure of the aneurysm since the operation, I found that pulsation had returned in it, and that a current of blood still continued through the sac. The force of pulsation would be represented by an estimate of about one-half of that previous to operation. The early reappearance of a pulse in the radial and superficial temporal arteries had led me to expect this, and rendered it probable that, in spite of the apparent tightness with which the ligature was drawn, a chink existed between the folds of the crumpled-up artery through which the direct current continued.-31st: Wound dressed; thoroughly aseptic. Pulse 96; temperature normal; respiration 20 . I may say now that this condition was maintained throughout, the patient kept absolutely free from fever or constitutional disturbance, and the wound aseptic till the final hæmorrhages necessitated a discontinuance of the dressings; these were changed about every third day. The pulsation in the aneurysm was slightly less than on the previous occasion, and in the radial artery was still further diminished. I hoped to favour the process of consolidation and cure of the aneurysm by immobilising the arm to the side, but though his pain and suffering were materially better than before operation, he was rather intolerant of this, and 


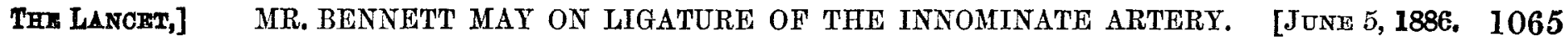

it could not be carried out very effectually.-April 5th: Has had rather more pain again, for which the use of morphia was resumed. Aneurysm harder and smaller. Pulsation distinctly less; radial pulse almost imperceptible; temporal pulse increasing.--8th: Aneurysm still harder and smaller. There appears now less doubt about the object for which the operation was undertaken being accomplished if he should recover from its effects.-10th: A stain of blood looking a day or so old was seen for the first time on changing the dressings.-11th: Late at night a sharp hæmorrhage occurred, which was stopped by plugging the wound.12th: Several small hæmorrhages took place every few hours, and were controlled by pressure. Patient kept well ander morphia. - 13th: Late at night another general hæmorrhage.-14th: Died in the evening.

Necropsy. ${ }^{2}$ - Part of the incision was firmly cicatrised; the unhealed part opened into a cavity containing clot. The walls of this cavity were well consolidated, and at the bottom was the artery, on which the knot of the ligature could be felt. The aneurysm was of much larger dimensions than appeared on the surface; it completely filled the upper aperture of the thorax, bulging into this cavity; it lay against the spinal column, and had absorbed and eroded almost quite across the bodies of several of the vertebræ; it was quite inseparable from the column. The cords of the brachial plexus were flattened out behind the tumour; the internal jugular and subclavian veins were collapsed and Hattened in front of it. The aneurysm sprang from the upper and back part of the artery. The mouth of the sac was very large, extending from within half an inch of the commencement nearly to the termination of the artery, so that the whole of the second, about half of the first, and a considerable portion of the third stages of the vessel were occupied by it. All the branches of the artery were in this area and opened into the aneurysmal sac. The remainder of the first portion of the vessel was of large size and flattened out in tront of the sac, to which it was adherent. The aneurysm contained a large central mass of passive clot. Some portions were lined with old fibrinous layers to the depth of a quarter of an inch, and within these and also incorporated with other parts of the sac wall was a less firm coagulum. Elsewhere, and particularly near the large mouths of entering vessels, the interior appeared as a serous ining. The arch of the aorta and innominate were vessels of large dimensions, but without local or fusiform expansion. They contrasted markedly with the exceptionally small size of the left common carotid and subclavian and of the right subclavian beyond the aneurysm; the latter was much reduced in size. The aorta was thickened and atheromatous ; the innominate also thickened and fibroid to a less degree. The position of the ligature was marked by a deep constriction almost exactly in its centre; the structures over the ligature were solid and organised, except at the knot, which was exposed; there was no purulent infiltration. The ligature still retained a firm hold on the vessel; one or two of the threads were partially absorbed and softened, but others scarcely changed. The knot, unfortunately very large and hard, was quite unaltered. Under the knot, in the front of the vessel and in the line of a fold or bend of its wall, was the obvious source of the hæmorrhage, in the form of a ragged hole about the size of a small pea; this opened into the vessel on both sides of the ligature. The further appearances were interesting and instructive in view of the debatable questions surrounding the use of animal ligatures. The wall of the innominate was thickened on each side of the ligature, the thickness gradually decreasing away from it. iith the exception of the hole corresponding to the knot, no part of the arterial wall was injured or divided, though under the ligature itself the wall was thinner than elsewhere. The inner coats were intact. It was obvious that a small chink remained between the crumpled-up folds of the vessel, the remainder being occluded by adhesion of the inner coats. The chink was closed by a moderately firm clot; a similar clot, with conical end, extended along the distal side of the artery nearly to the bifurcation. The clot was only slightly adherent to the interior of the vessel, except under the ligature, where the adhesion was more firm. The hole in the wall of the artery having been closed, it was shown, by injecting water, that the vessel was wholly occluded at the seat of ligature. On the heart side there was a thin diaphragm of clot with a conica end, but it extended a very inconsiderable distance. 1. Specimen shown at the meeting of the Pathological Section of the
Birmingham and Midland Counties Branch of the British Medical Association, Nay 7 thl. the bulk of the hæmorrhage no doubt occurred here, some of the clot may have got carried out during life. The walls of the common carotid were thickened, its cavity much contracted, but free from clot. There was free communication between it and the subclavian.

The observations which I submit on this case will be very brief. Into the general history and merits of the operation I do not propose to enter. Mr. William Thompson has recently handled this part of the subject in a complete manner, ${ }^{2}$ and the grounds on which he defends and justifies the operation in suitable cases appear to me to be thoroughly sound. His own case, which is reported in his able and exhaustive paper, and which so nearly obtained success, goes far in support of them. I certainly do not think that, on the experience of the old methods of ligature, we are to regard the subject as a closed book not to be reopened. In dealing with this particular case I was not unmindful of the alternative courses. I had satisfied myself that medical treatment would be unavailing, while the severity of the patient's suffering rendered it most undesirable to abandon the case altogether. My reason for selecting this operation was simply its directness and certainty of action, and the belief that in it alone lay any hope of cure. I at once eliminated from consideration such methods of treatment as galvanopuncture and introduction of foreign material, on the ground that, from what I have read and seen of them, they are exceedingly fatal, while their value as a means of cure is almost problematical. Proximal pressure was impossible, the aneurysmal sac lying behind the first part of the artery. Amputation at the shoulder-joist needs, I think, more to recommend it than the hitherto limited experience of its use affords us before a surgeon will strongly urge such a serious mutilation on a patient. The distal ligature might have been tried, and could I have realised beforehand the condition of the artery it is quite likely this would have been the case. However, the vessel was fully exposed before I was aware of its state. There was no physical sign in the chest to reveal it, and the pulsation in the episternal notch was clearly that of the common carotid displaced by the tumour. Moreover, it appeared from the position of the tumour and the absence of a bruit that the mouth of the sac was of large size, and included just that portion of the vessel most occupied by large branches. Consequently I entertained but little hope of any backward development of a thrombus and a cure in this way. The post-mortem examination verifies the truth of this. When the artery was exposed the condition was obviously very unfavourable to success. I could see there would be increased difficulty in stopping pulsation and unusual risk of rupture of the artery, but I did not feel called upon to abandon the operation and close the wound, as so large a share of the operative risk had already been surmounted, and the vessel, though large in size, showed no local or fusiform expansion. I regarded the dilatation as in great part due to the resistance of the aneurysm beyond. The fact that I was unable to complete the ligature with the material originally intended may be matter for regret. The subsequent history and the postmortem appearances show that if a knot could be avoided a different result was possible, even on a vessel so unfavourable. But I confess that the case would scarcely have been a fair one as a test for the material, and I quite anticipate that Mr. Barwell would not contemplate its application to such a vessel. In explanation of the breakage, I think it fair to say that the ligature was not only submitted to an exceptionally severe strain, but had probably got damaged in its dry state. The use of silk, or of any material which, though having the advantage of unlimited strength, must necessarily cut through the vessel and be cast off by ulceration, was out of the question. In employing catgut, sufficient had to be used to bear a good strain in tightening, to last a considerable time without absorption, and to maintain the integrity of the arterial wall. To gain all these advantages I used a cord of five or six medium-sized. threads laid side by side, and as a material like this requires very careful tying to secure it, I felt it prudent to make a third knot. This knot, lying on a fold of the vessel, was, by its hardness and resistance to the forcible pulsation, the immediate cause of rupture of the artery.

In undertaking the operation, I entertained the greatest confidence, as I do now, in the value of the animal absorbable material for ligature, of the strict antiseptic condition of the wound, and in the principle of maintaining the integrity of 
the arterial wall by the flat ligature so ably advocated by Mr. Barwell. It is on the latter of these points that opinion seems to be most divided. Should the arterial coats be cut through or not? I do not think we need seek to give one uniform answer to this question for all cases, but that it may wisely vary for different circumstances. With vessels of secondary size, in positions where the strict antiseptic condition of the wound is pretty sure, and, above all, where early deep union of the wound can be effected, a simple round cord of absorbable material (ordinary catgut), tied so as to divide the walls, leaves little room for improvement. There is in the latter point an additional security for permanent occlusion of the vessel. But it is well known that if the wound inflames and suppurates, the advantages of the animal ligature may be forfeited by solution of continuity of the vessel. It is to be recognised that the wound after ligature of the innominate is ill adapted for early union, and difficult to keep antiseptic. Though I was successful in securing the latter point in this patient, I found that the bony framework of the chest prevented deep apposition of the parts, and that a considerable cavity resulted in spite of graduated and elastic pressure applied to that end, as recommended by Mr. Thompson in the paper referred to. There can be little doubt, therefore, about the importance of the principle of maintaining the integrity of the arterial wall by the flat ligature in the case of the innominate artery. That an artery ligatured in this way becomes permanently occluded has been amply proved by Mr. Barwell's cases, and by those of Lister, Thompson, and others; it also seems that the existence of a chink at the point of ligature permitting a direct current of blood is no bar to the process. On this latter point I think the postmortem appearances in my case bear in an interesting manner. They show that such a process of occlusion was in actual progress, though to an inconsiderable degree. When I found that pulsation had returned in the sac and in the radial and temporal arteries, I inferred that this was wholly due to the direct current past the ligature, and I seriously entertained the idea of reopening the wound and replacing it. Then, as the pulsation in the sac and the radial subsequently diminished, it appeared as if occlusion were gradually being effected by coagulation at the ligature, and there seemed increasing likelihood of the aneurysm being cured if the operation were survived. But the pulsation was still going on at a time when, as the necropsy shows, there could be no direct current past the ligature, so that it appears as if a good deal of this pulsation was due to the current of blood into the sac from the mouths of the large vessels opening there and from the common carotid. This fact, with the comparatively little change effected in the aneurysm, leave it still open to doubt whether the object for which the operation was undertaken would have been accomplished had the patient lived. The collateral circulation might have proved too direct and free.

\section{ABSCESS OF THE SPLEEN OCCURRING IN ENTERIC FEVER.}

BY ROBERT S. ARCHER, B.A., M.B.

PHYSICIAY TO THE NETHERFIELD FEVER HOSPITAL, LIVERPOOL.

ON assuming duty at Netherfield Fever Hospital towards the end of last October, I found a boy, aged fourteen years, suffering from what appeared to be an intercurrent relapse or recrudescence of enteric fever. He had been admitted to hospital on Oct. 5 th, 1885, under the care of my colleague, Dr. Dunbar, with a history of illness of two days duration, which commenced with anorexia, vomiting, and rigors. His throat is reported to have been sore, and the tonsils superficially ulcerated. $\mathrm{He}$, as far as 1 could ascertain, presented none of the common signs or symptoms of enteric fever; in fact, I believe he was sent to hospital as suffering from scarlatina, which was doubtless owing to the slight throat symptoms and a doubtful evanescent red rash. The temperature, which had been febrile since his admission, became normal on Oct. 18th, or about the sixteenth day of apparent illness. It remained normal until the 2lst, when it again became febrile, and remained so till the patient's death on Nov. 1st. Oct. 24 th was marked by slight pain 1885 . along the course of the lower ribs on the left side, which was thought might have been due to pericarditis or pleuritis. However, careful examination failed to detect the existence of either of these conditions. On Oct. $26 \mathrm{th}$ the left hypochondriac pain became more marked, and on the evening of the following day there was slight general abdominal tenderness. The next twenty-four hours was marked by vomiting, intensification of the abdominal tenderness, and increased tympanitis, especially manifest along the course of the transverse colon. All these signs became more painfully apparent during the next three days, and there was superadded a certain amount of stupor and low muttering delirium. The patient died early on the morning of Nor. 1st apparently from peritonitis, due, as was thought, solely to perforation of the intestine.

At the necropsy (made about ten hours after death) there was fairly marked rigor mortis and considerable emaciation. The abdomen was generally and equally distended, and there was distinct general purulent peritonitis. The omentum and coils of the intestines were loosely held together by layers of yellow puriform lymph. A large quantity of yellow fluid, which had evidently escaped from the intestines, occupied the depending portions of the abdominal and pelvic cavities. The intestines contained some yellow semi-solid fæces. There was a perforation situater about a foot and a half from the ileo-cæcal valve, occupying the base of a cicatrising ulcer. Several of Peyer's patches were observed to be ulcerated, quite free from sloughs, and evidently in process of cicatrisation. The long axis of the ulcers was usually transverse to the course of the intestine, owing, I suppose, to the fact that cicatrisation had commenced from each end, and not laterally. There was a mass of almost entirely cicatrised ulceration in the neighbourhood of the ileo-cæcal valve. The liver was enlarged and pale. The spleen was enlarged, and generally soft and congested. Its convex surface was covered by a more or less uniform layer of yellow puriform lymph. In its upper part, and under the surface of its external aspect, was an abscess which would contain a large-sized walnut. This collection of pus had ruptured into the peritoneal cavity. The lungs were found to be hypostatically congested, but otherwise healthy. The pericardium contained a considerable quantity of clear, straw-coloured fluid. The heart was healthy.

Remarks.-Abscess of the spleen, a rare affection under all circumstances, would appear to be, contrary to what might be expected when we have regard to the fact that this organ is always affected to a greater or less degree in enteric fever, of very infrequent occurrence in this disease. Murchison's vast experience gives only two cases, both of which died, in what appeared to be a relapse, from genera peritonitis, and he mentions five others from other authors. A considerable number of necropsies on the bodies of patients dying of enteric fever at Netherfield Fever Hospita? during the last five years supplies only this single example of splenic abscess. In November, 1883, Dr. Coates showed at the Calcutta Medical Society a boy aged four with enlargement of the spleen, the result of malarious fever. An incision was made into an abscess, and about ten ounces of pus discharged. ${ }^{2}$ As regards diagnosis, it may be mentioned that in this case the actual condition of affairs, as far as the spleen was concerned, was not determined clinically. But if in another case there were presented for our observation tenderness in the region of the spleen, pain under the left ribs, and a "creeping" peritonitis gradually extending from this as a centre over the abdomen, the existence of splenic abscess would be suggested to the mind; and, further. that the abscess had burst and set up general inflammation of the serous membrane would be quite a reasonable conclusion to arrive at. Although the boy was reported to have been taken ill only two days before admission to hospital, judging from the condition of the ulcers I am strongly inclined to believe he must have been going about with the fever on him for at least a week before the apparent onset of the disease. The reappearance of febrile symptoms after an apyrexial state of three days cannot be regarded as a relapse in the true sense of the word, but must be looked upon rather as symptomatic of the splenic mischief, just as in this disease, as well as in any other of the specific fevers, a continuance of the fever beyond the usual time, or a reappearance of fever, accompanied by manifest local lesions, must be regarded as purely symptomatic. 